Journal of Animal and Veterinary Advances 11 (2): 252-259, 2012

ISSN: $1680-5593$

(C) Medwell Journals, 2012

\title{
Molecular Cloning, Sequence Identification and Tissue Expression Profile of Three Novel Sheep (Ovis aries) Genes-RHOA, RCHY1 and $R S U 1$
}

\author{
${ }^{1}$ Liu Yonggang and ${ }^{2}$ Xia Xueshan \\ ${ }^{1}$ College of Animal Science and Technology, \\ Yunnan Agricultural University, 650201 Kunming, China \\ ${ }^{2}$ Faculty of Life Science and Technology, \\ Kunming University of Science and Technology, 650224 Kunming, China
}

\begin{abstract}
The complete coding sequences of three sheep genes-RHOA, RCHY1 and RSU1 were amplified using the Reverse Transcriptase-Polymerase Chain Reaction (RT-PCR). The sheep RHOA gene encodes a protein of 193 amino acids that shares high homology with the ras homolog gene family, member A (RHOA) proteins of fifteen species- cattle (100\%), rat (99\%), human (100\%), mouse (99\%), chicken (99\%), dog (99\%), turkey (99\%), sumatran orangutan (99\%), poephila guttata (99\%), Atlantic salmon $(97 \%)$, green anole $(96 \%)$, rainbow trout (96\%), African clawed frog (96\%), zebrafish (96\%) and Western clawed frog (95\%). The sheep RCHY1 gene encodes a protein of 261 amino acids that shares high homology with the ring finger and CHY zinc finger domain containing 1 (RCHY1) proteins of nine species-cattle (94\%), giant panda (92\%), pig (91\%), human (90\%), white-tufted-ear marmoset $(90 \%)$, chimpanzee $(90 \%)$, rabbit $(89 \%)$, mouse $(86 \%)$ and rat $(84 \%)$. The sheep RSU1 gene encodes a protein of 277 amino acids that shares high homology with the ras suppressor protein 1 (RSU1) proteins of twelve species-cattle (99\%), chimpanzee $(98 \%)$, dog $(99 \%)$, human (98\%), giant panda (98\%), horse (98\%), rabbit (98\%), chicken (96\%), mouse (97\%), African clawed frog (94\%), Western-clawed-frog (94\%) and zebrafish (91\%). Finally, these three novel sheep genes were assigned to GeneIDs: 100302078 , 100302546 and 100302548 . The phylogenetic analysis revealed that the sheep RHOA, RCHY1 and RSU1 genes all have a closer genetic relationship with the $R H O A, R C H Y 1$ and RSU1 genes of cattle. Tissue expression profile analysis demonstrated that sheep $R H O A, R C H Y 1$ and $R S U 1$ genes were all generally but differentially expressed in detected tissues.
\end{abstract}

Key words: Sheep, RHOA, RCHY1, RSU1, tissue expression, China

\section{INTRODUCTION}

Ras homolog gene family, member A (RHOA) belongs to the RhoA-like sub-family. The RhoA-like sub-family consists of RHOA, RHOB and RHOC. Latest researches demonstrated that $\mathrm{RHOA}$ promotes the formation of stress fibers and focal adhesions, regulating cell shape, attachment and motility ( $\mathrm{Su}$ et al., 2006). RHOA is vital for muscle contraction in vascular smooth muscle cells, RHOA plays a key role in cell contraction, differentiation, migration and proliferation (Peyton et al., 2008). RHOA activities appear to be elaborately regulated in a time- and space-dependent manner to control cytoskeletal changes (Maekawa et al., 1999; Ohta et al., 1999; Saeki et al., 2005). Ring finger and CHY zinc finger domain containing 1 ( $\mathrm{RCHY} 1$ ), a member of proteins containing the ring finger and $\mathrm{CHY}$ zinc finger domain has ubiquitin ligase activity. It mediates E3-dependent ubiquitination and proteasomal degradation of target proteins including TP53, HDACl and CDKN1B thus, regulating their levels and cell cycle progression (Wu et al., 2010; Yan et al., 2010; Shloush et al., 2011).

Ras Suppressor protein 1 (RSU1) gene encodes a protein that is involved in the ras signal transduction pathway, growth inhibition and nerve-growth factor induced differentiation processes as determined in mouse and human cell line studies. In mouse, the encoded protein was initially isolated based on its ability to inhibit v-Ras transformation. Multiple alternatively spliced transcript variants for this gene have been reported; one of these variants was found only in glioma tumors (Chunduru et al., 2002; Dougherty et al., 2005, 2008).

As mentioned before, RHOA, RCHY1 and RSU1 genes are three genes which have important functions. Until today, RHOA, RCHY1 and RSU1 genes had been reported in human and other animals but the sheep $R H O A$,

Corresponding Author: Xia Xueshan, Faculty of Life Science and Technology, Kunming University of Science and Technology, 650224 Kunming, China 
RCHY 1 and RSU1 genes have not been reported yet. In present experiment, the researchers will isolate the coding sequences of sheep $R H O A, R C H Y 1$ and RSU1 genes based on the coding sequence information of RHOA, RCHY1 and RSU1 genes from human or other mammals and their highly homologous sheep ESTs sequence information, subsequently perform sequence and tissue expression profile analysis for these genes. These will establish the primary foundation of understanding these three sheep genes.

\section{MATERIALS AND METHODS}

Animals and sample preparation: Five adult Yunnan local sheep were slaughtered. Spleen, skin, lung, fat, muscle, heart, liver, kidney and ovary samples were collected, frozen in liquid nitrogen and then stored at $-80^{\circ} \mathrm{C}$. The total RNA was extracted using the total RNA extraction kit (Gibco, USA). First-strand cDNA synthesis was performed as that described by Liu et al. (2004). These first-strand cDNA samples were used to perform RT-PCR for the isolation of sheep RHOA, RCHY1 and RSU1 genes and for the tissue expression profile analysis.

Isolation of the sheep $R H O A, R C H Y 1$ and $R S U 1$ genes: The primers for sheep RHOA gene isolation were designed based on the coding sequence information of human RHOA gene and its highly homologous sheep EST sequences: DY503081 andEE758341. Similarly, the primers for sheep RCHY1 gene isolation were designed based on the coding sequence information from human RCHY1 gene and its highly homologous sheep EST sequences; DY519128 and EE754481. The primers for sheep RSU1 gene isolation were designed based on the coding sequence information from human and mouse RSU1 genes and their highly homologous sheep EST sequences; DY495018 and FE027712. These primer sequences and their annealing temperature for RT-PCR reaction were shown in Table 1 . The RT-PCR was performed to isolate these three sheep genes using the pooled cDNAs from different tissues above. The $25 \mu \mathrm{L}$ reaction system was; $2.0 \mu \mathrm{L}$ cDNA, $2.5 \mu \mathrm{L} 2 \mathrm{mM}$ mixed dNTPs, $2.5 \mu \mathrm{L} 10 \times$ Taq DNA polymerase buffer, $2.5 \mu \mathrm{L}$ $25 \mathrm{mM} \mathrm{MgCl}_{2}, 2.0 \mu \mathrm{L} 10 \mu \mathrm{M}$ forward primer, $2.0 \mu \mathrm{L} 10 \mu \mathrm{M}$ reverse primer, 2.0 units of Taq DNA polymerase (1 U/1 $\mu \mathrm{L}$ ) and $9.5 \mu \mathrm{L}$ sterile water. The PCR program initially started with a $94^{\circ} \mathrm{C}$ denaturation for $4 \mathrm{~min}$ followed by 35 cycles of $94^{\circ} \mathrm{C} / 50 \mathrm{sec}, \mathrm{Ta}^{\circ} \mathrm{C} / 50 \mathrm{sec}$, $72^{\circ} \mathrm{C} / 50 \mathrm{sec}$ then $72^{\circ} \mathrm{C}$ extension for $10 \mathrm{~min}$, finally $4^{\circ} \mathrm{C}$ to terminate the reaction (Table 1 ).

These PCR products for sheep RHOA, RCHY1 and RSU1 genes were then cloned into PMD18-T vector and sequenced bidirectionally with the commercial fluorometric method. At least five independent clones were sequenced for every gene.
Table 1: Primers for sheep RHOA, RCHYl, RSUl and beta-actin genes and their annealing Temperature ( $\mathrm{Ta})$

\begin{tabular}{llc}
\hline Genes & Primer sequence & $\mathrm{Ta} /{ }^{\circ} \mathrm{C}$ \\
\hline RHOA & Forward 5'-ATGGCTGCCATCCGGAAG-3' & \\
& Reverse: 5'-TCACAAGACAAGGCACCCAG-3' & 61 \\
$R C H Y I$ & Forward 5'-ATGGCTTCCTCGACGCTG-3' & \\
& Reverse: 5'-TCATTGCTGATCTAATGAATTT-3' & 61 \\
RSUl & Forward: 5'-ATGTCCAAGTCTCTGAAA-3' & \\
& Reverse: 5'-TTATTTATTCTTGGCTGCC-3' & 52 \\
Beta-actin & Forward: 5'-CTTGATGTCACGGACGATTT-3' & \\
& Reverse: 5'-CACGGCATTGTCACCAACT-3' & 56 \\
\hline
\end{tabular}

RT-PCR for tissue expression profile analysis: RT-PCR for tissue expression profile analysis was performed as previously described elsewhere (Liu and Gao, 2009; Yonggang and Shizheng, 2009; Liu, 2009). The researchers selected the housekeeping gene beta-actin (Accession No.: NM_001009784) as a positive control. The primers of sheep $R H O A, R C H Y 1$ and $R S U 1$ genes which were used to perform the RT-PCR for tissue expression profile analysis were same as the primers for isolation RT-PCR above. The PCR reactions were optimized for a number of cycles to ensure product intensity within the linear phase of amplification. The $25 \mu \mathrm{L}$ reaction system was: $1 \mu \mathrm{L}$ cDNA (100 ng $\left.\mu \mathrm{L}^{-1}\right), 5$ pmoles each oligonucleotide primer, $2.5 \mu \mathrm{L}, 2 \mathrm{mmol} \mathrm{L}^{-1}$ mixed dNTPs, $2.5 \mu \mathrm{L} 10 \times$ Taq DNA polymerase buffer, $2.5 \mu \mathrm{L} 25 \mathrm{mmol} \mathrm{L}{ }^{-1} \mathrm{MgCl}_{2}$, 1.0 unit of Taq DNA polymerase and finally add sterile water to volume $25 \mu \mathrm{L}$. The PCR program initially started with a $94^{\circ} \mathrm{C}$ denaturation for $4 \mathrm{~min}$ followed by 25 cycles of $94^{\circ} \mathrm{C} / 50 \mathrm{sec}, \mathrm{Ta}^{\circ} \mathrm{C} / 50 \mathrm{sec}, 72^{\circ} \mathrm{C} / 50$ sec then $72^{\circ} \mathrm{C}$ extension for $10 \mathrm{~min}$, finally $4^{\circ} \mathrm{C}$ to terminate the reaction (Table 1).

Sequence analysis: The cDNA sequence prediction was conducted using GenScan software (http://genes.mit.edu/ GENSCAN.html). The protein prediction and analysis were performed using BLAST tool at the National Center for Biotechnology Information (NCBI) server (http://www. ncbi.nlm.nih.gov/BLAST) and the ClustalW software (http://www.ebi.ac.uk/clustalw).

\section{RESULTS AND DISCUSSION}

RT-PCR results for sheep $R H O A, R C H Y 1$ and $R S U 1$ genes: Through RT-PCR with pooled tissue cDNAs, for sheep RHOA, RCHY1 and RSU1 genes, the resulting PCR products were 582,786 and 834 bp (Fig. 1).

Sequence analysis: These cDNA nucleotide sequence analysis using the BLAST software at NCBI server (http://www.ncbi.nlm.nih.gov/BLAST) revealed that these three genes were not homologous to any of the known sheep genes and they were then deposited into the GenBank database (Accession No.: FJ943984, FJ937958 and FJ937963). The sequence prediction was carried out 

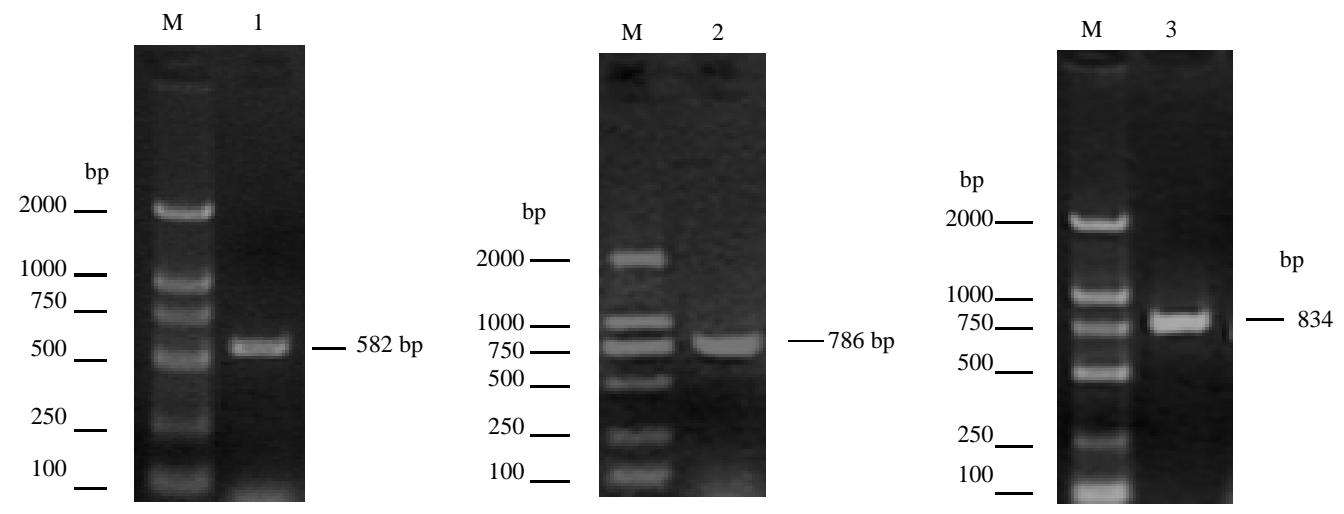

Fig. 1: RT-PCR results for sheep RHOA, RCHY1 and RSU1 genes. M: DL 2000 DNA markers; 1: PCR product for sheep $R H O A$ gene; 2: PCR product for sheep RCHY1 gene; 3: PCR product for sheep RSU1 gene

using the GenScan software and results showed that the 582,786 and 834 bp cDNA sequences represent three single genes which encoded 193, 261 and 277 amino acids, respectively. Finally, these three novel sheep genes were assigned to GeneIDs; 100302078, 100302546 and 100302548 .

Further BLAST analysis of these proteins revealed that the sheep RHOA protein has high homology with the ras homolog gene family, member $\mathrm{A}$ (RHOA) proteins of fifteen species-cattle (Accession No.: NP_788818; 100\%), rat (Accession No.: NP_476473; 99\%), human (Accession No.: NP_001655; 100\%), mouse (Accession No.: NP_058082; 99\%), chicken (Accession No.: NP_990035; 99\%), dog (Accession No.: NP_001003273; 99\%), Turkey (Accession No.: ADX97247; 99\%) sumatran orangutan (Accession No.: NP_001124755; 99\%), poephila guttata (Accession No: ACH45076; 99\%), Atlantic salmon (Accession No.: ACI33725; 97\%), green anole (Accession No.: XP_003216698; 96\%), rainbow trout (Accession No.: NP_001158532; 96\%), African clawed frog (Accession No.: AD40671; 96\%), zebrafish (Accession No.: NP_998515; 96\%) and Western clawed frog (Accession No.: CAJ81715; 95\%) (Fig. 2).

The sheep RCHY1 protein has high homology with the ring finger and $\mathrm{CHY}$ zinc finger domain containing 1 (RCHY1) proteins of nine species-cattle (Accession No.: NP_001077223; 94\%), giant panda (Accession No.: XP_002919200; 92\%), pig (AccessionNo.:NP_001090959; 91\%), human (Accession No.: NP_056251; 90\%), white-tufted-ear marmoset (Accession No: XP_002745761; 90\%), chimpanzee (Accession No.: XP_517222; 90\%), rabbit (Accession No.: XP_002717057; 89\%), mouse (Accession No.: NP_0808337; 86\%) and rat (Accession No.: NP_001007619; 84\%) (Fig. 3).
The sheep RSU1 protein has high homology with the ras suppressor protein 1 (RSU1) proteins of twelve species-cattle (Accession No.: NP_001035691; 99\%), chimpanzee (Accession No.: XP_001151460; 98\%), dog (Accession No.: XP 535177; 99\%), human (Accession No.: NP_036557; 98\%), giant panda (Accession No.: XP_002918673; 98\%), horse (Accession No.: XP_001498103; 98\%), rabbit (Accession No: XP_002717469; 98\%), chicken (Accession No:: NP_001 186520; 96\%), mouse (AccessionNo.: NP_033131; 97\%), African clawed frog (Accession No.: NP_001085943; 94\%), Western-clawed-frog (Accession No.: NP_001015695; 94\%) and zebrafish (Accession No.: XP_002666722; 91\%) (Fig. 4).

Based on the results of the alignment of RHOA, RCHY1 and RSU1 proteins, three phylogenetic trees were constructed using the Dendrogram procedure of ClustalW software as shown in Fig. 5-7. The phylogenetic analysis revealed that the sheep $R H O A, R C H Y 1$ and $R S U 1$ genes all have a closer genetic relationship with the $R H O A$, $R C H Y 1$ and RSU1 genes of cattle.

Tissue expression profile: Tissue expression profile analysis was carried out and results revealed that the sheep RHOA, RCHY1 and RSU1 genes are all generally but differentially expressed in tissues including spleen, lung, muscle, kidney, ovary, skin, liver, heart and fat (Fig. 8).

In the current study, the researchers firstly get the coding sequences of sheep RHOA, RCHY1 and RSU1 genes by RT-PCR. With the development of modern bioinformatics, establishment of specific sheep NCBI EST database and different convenient analysis tools, researchers can easily find the useful ESTs which were highly homologous to the coding sequences of human 


Rat
Mouse
Sheep
Cattle
Human
Sumatran orangutan
Dog
Poephila guttata
Chicken
Turkey
Zebrafish
Green anole
Rainbow trout
Atlantic salmon
African clawed frog
Western clawed frog

Rat

Mouse

Sheep

Cattle

Human

Sumatran orangutan

Dog

Poephila guttata

Chicken

Turkey

Zebrafish

Green anole

Rainbow trout

Atlantic salmon

African clawed frog

Western clawed frog

Rat

Mouse

Sheep

Cattle

Human

Sumatran orangutan

Dog

Poephila guttata

Chicken

Turkey

Zebrafish

Green anole

Rainbow trout

Atlantic salmon

African clawed frog

Western clawed frog

Rat

Mouse

Sheep

Cattle

Human

Sumatran orangutan

Dog

Poephila guttata

Chicken

Turkey

Zebrafish

Green anole

Rainbow trout

Atlantic salmon

African clawed frog

Western clawed frog
MAAIRKKLVIVGDGACGKTCLLIVFSKDQF PEVYVPTVFENYVADIEVDGKQVELALWDT MAAIRKKLVIVGDGACGKTCLLIVFSKDQF PEVYVPTVFENYVADIEVDGKQVELALWDT MAAIRKKLVIVGDGACGKTCLLIVFSKDQF PEVYVPTVFENYVADIEVDGKQVELALWDT MAAIRKKLVIVGDGACGKTCLLIVFSKDQF PEVYVPTVFENYVADIEVDGKQVELALWDT MAAIRKKLVIVGDGACGKTCLLIVFSKDQF PEVYVPTVFENYVADIEVDGKQVELALWDT MAAIRKKLVIVGDGACGKTCLLIVFSKDQF PEVYVPTVFENYVADIEVDGKQVELALWDT MAAIRKKLVIVGDGACGKTCLLIVFSKDQFPEVYVPTVFENYVADIEVDGKQVELALWDT MAAIRKKLVIVGDGACGKTCLLIVFSKDQFPEVYVPTVFENYVADIEVDGKQVELALWDT MAAIRKKLVIVGDGACGKTCLLIVFSKDQF PEVYVPTVFENYVADIEVDGKQVELALWDT MAAIRKKLVIVGDGACGKTCLLIVFSKDQF PEVYVPTVFENYVADIEVDGKQVELALWDT MAAIRKKLVIVGDGACGKTCLIIVFSKDQF PEVYVPTVFENYVADIEVDSKQVELALWDT MAAIRKKLVIVGDGACGKTCLLIVFSKDQF PEVYVPTVFENYVADIEVDGKQVELALWDT MAAIRKKLVIVGDGACGKTCLLIVFSKDQFPEVYVPTVFENYVADIEVDSKQVELALWDT MAAIRKKLVIVGDGACGKTCLLIVFSKDQF PEVYVPTVFENYVADIEVDSKQVELALWDT MAAIRKKLVIVGDGACGKTCLLIVFSKDQFPEVYVPTVFENYVADIEVDGKQVELALWDT MAAIRKKLVIVGDGACGKTCLLIVFSKDQF PEVYVPTVFENYVADIEVDSKQVELALWDT $* * * * * * * * * * * * * * * * * * * * * * * * * * * * * * * * * * * * * * * * * * * * * * * * * * * * * * * . * * * * * * * * * * *$

AGQEDYDRLRPLSYPDTDVILMCFSIDSPDSLENI PEKWT PEVKHFCPNVPI ILVGNKKD AGQEDYDRLRPLSYPDT DVILMCFSIDSPDSLENI PEKWT PEVKHFCPNVPI ILVGNKKD AGQEDYDRLRPLSYPDT DVILMCFS IDSPDSLENI PEKWT PEVKHFCPNVPI I LVGNKKD AGQEDYDRLRPLSYPDTDVILMCFSIDSPDSLENI PEKWT PEVKHFCPNVPI ILVGNKKD AGQEDYDRLRPLSYPDTDVILMCFSIDSPDSLENI PEKWT PEVKHFCPNVPI ILVGNKKD AGQEDYDRLRPLSYPDTDVILMCFSIDSPDSLENI PEKWT PEVKHFCPNVPI ILVGNKKD AGQEDYDRLRPLSYPDT DVILMCFSIDSPDSLENI PEKWT PEVKHFCPNVPI ILVGNKKD AGQEDYDRLRPLSYPDTDVILMCFSIDSPDSLQNI PEKWT PEVKHFCPNVPI ILVGNKKD AGQEDYDRLRPLSYPDTDVILMCFSIDSPDSLENI PEKWT PEVKHFCPNVPI ILVGNKKD AGQEDYDRLRPLSYPDT DVILMCFSIDSPDSLENI PEKWT PEVKHFCPNVPI ILVGNKKD AGQEDYDRLRPLSYPDTDVILMCFSIDSPDSLENI PEKWT PEVKHFCPNVPI ILVGNKKD AGQEDYDRLRPLSYPDT DVILMCFSIDSPDSLENI PEKWT PEVKHFCPNVPI ILVGNKKD AGQEDYDRLRPLSYPDT DVILMCFSIDSPDSLENI PEKWT PEVKHFCPNVPI ILVGNKKD AGQEDYDRLRPLSYPDTDVILMCFSIDSPDSLENI PEKWT PEVKHFCPNVPI ILVGNKKD AGQEDYDRLRPLSYPDT DVILMCFSIDSPDSLENI PEKWT PEVKHFCPNVPI ILVGNKKD AGQEDYDRLRPLSYPDTDVILMCFSIDSPDSLENI PEKWT PEVKHFCPNVPI ILVGNKKD

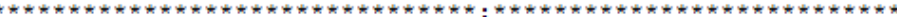
LRNDEHTRRELAKMKQE PVKPEEGRDMANRIGAFGYMECSAKTKDGVREVFEMATRAALQ LRNDEHTRRELAKMMQE PVKPEEGRDMANRI GAFGYMECSAKTKDGVREVFEMATRAALQ LRNDEHTRRELAKMKQE PVKPEEGRDMANRIGAFGYMECSAKTKDGVREVFEMATRAALQ LRNDEHTRRELAKMKQE PVKPEEGRDMANRIGAFGYMECSAKTKDGVREVFEMATRAALQ LRNDEHTRRE LAKMMKOE PVKPEE GRDMANRI GAFGYMECSAKTKDGVREVFEMATRAALQ LRNDEHTRREIAKMMKQE PVKPEEGRDMANRIGAFGYMECSAKTKDGVREVFEMATRAAL $Q$ LRNDEHTRRE LAKMKQE PVKPTEGRDMANRIGAFGYMECSAKTKDGVREVFEMATRAAL LRNDEHTRRELAKMKQE PVKPEEGRDMANRI GAFGYMECSAKTKDGVREVFEMATRAALQ LRNDEHTRRELAKMKQE PVKPEEGRDMANRIGAFGYMECSAKTKDGVREVFEMATRAAL $Q$ LRNDEHTRRELAKMKQE PVKPEEGRDMANRIGAFGYMECSAKTKDGVREVFEMATRAALQ LRNDEHTRRELAKMMQE PVKPEEGRDMANRINAFGYLECSAKTKDGVREVFEMATRAALQ LRNDEHTRRELAKMKQE PVKPEEGRDMANRI GAFGYLECSAKTKDGVREVFEMATRAALQ LRNDE HTRRELAKMMKQE PVKPEE GRDMANRI SAFGYMECSAKTKDGVREVFEMATRAALQ LRNDEHTRRELAKMKQE PVKPEEGRDMANRI GAFGYMECSAKSKDGVREVFEMATRAALQ LRNDEHTRRELTKMKQE PVKPEEGRDMANRISAYAYMECSAKTKDGVREVFELATRAALQ LRNDEHTRRELTKMKQE PVKPEEGRDMANRISAYGYMECSAKTKDGVREVFELATRAALQ $* * * * * * * * * * *: * * * * * * * * * * * * * * * * * * * . *::_{*}^{*}: * * * * *: * * * * * * * * *: * * * * * * *$

ARRGKKKSGCLII ARRGKKKSGCLII ARRGKKKSGCLVL ARRGKKKSGCLVI ARRGKKKSGCLVI ARRGKKKSGRLVI ARRGKKKSGCLVI ARRGKKKSGCLFI ARRGKKKSGCLII ARRGRKKSGCLII ARKRGKKSGCLII AKRGRKKTSCQLI ARRGKPRNKCLLI ARRGKKSNKCLLI ARRGKKKPRCLLI ARRGKKKTTCLII *: :

Fig. 2: The alignment of the protein encoded by sheep $R H O A$ gene and fifteen other kinds RHOA proteins 
Human

White-tufted-ear marmoset

Chimpanzee

Sheep

Cattle

Pig

Giant panda

Rabbit

Mouse

Rat

Human

White-tufted-ear marmoset

Chimpanzee

Sheep

Cattle

Pig

Giant panda

Rabbit

Mouse

Rat

Human

White-tufted-ear marmoset

Chimpanzee

Sheep

Cattle

Pig

Giant panda

Rabbit

Mouse

Rat

Human

White-tufted-ear marmoset

Chimpanzee

Sheep

Cattle

Pig

Giant panda

Rabbit

Mouse

Rat

Human

White-tufted-ear marmoset

Chimpanzee

Sheep

Cattle

Pig

Giant panda

Rabbit

Mouse

Rat

Human

White-tufted-ear marmoset

Chimpanzee

Sheep

Cattle

Pig

Giant panda

Rabbit

Mouse

Rat
MAATAREDGASGQERGQRGCEHYDRGCLLKAPCCDKLYTCRLCHDNNEDH MAATAREDGASGQERVQRGCEHYDRGCLLKAPCCDKLYTCRLCHDNNEDH MAATAREDGAGGQERGQRGCEHYDRGCLLKAPCCDKLYTCRLCHDNNEDH MASSTLEDGARGQAQRRRGCEHYDRGCLLKAPCCDKLYTCRLCHDNNEDH MAFSALEDGARGQAQRRRGCEHYDRGCLLKAPCCDKLYTCRLCHDNNEDH MAASAREDGGRGQEHGRRGCEHYDRGCLLKAPCCDKLYTCRLCHDSNEDH MA.A.SAREDGARSQKQGRRGCEHYDRGCLLKAPCCDKLYTCRLCHDNNEDH MAATAREDGARSRQRGQRGCEHYDRGCLLKAPCCDKLYTCRLCHDNNEDH MAATAREDGVRNLAQGPRGCEHYDRACLLKAPCCDKLYTCRLCHDTNEDH MAATAQEDGVRSPAPGPRGCEHYDRACLLKAPCCDKLYTCRLCHDTHEDH $* *:$ : $* * *$. $\quad * * * * * * * * . * * * * * * * * * * * * * * * * * * * .: * * *$

QLDRFKVKEVQCINCEKIQHAQQTCEECSTLFGEYYCDICHLFDKDKKQY QLDRFKVKEVQCINCEKIQHAQQTCEECSTLFGEYYCDICHLFDKDKKQY QLDRFKVKEVQCINCEKIQHAQQTCEECSTLFGEYYCDICHLFDKDKKQY QLDRFKVKEVQCINCEKIQRAQQTCEECSTLFGEYYCSVCHLFDKDKKQY QLDRFKVKEVQCINCEKIQHAQQTCEECSTLFGEYYCSVCHLFDKDKKQY QLDRFKVKEVQCINCEKIQHAQQICEECSTLFGEYYCSICHLFDKDKKQY QLDRFKVKEVQCINCEKIQHAQQTCEECSTLFGEYYCS ICHLFDKDKKQY QLDRFKVKEVQCINCEKIQHAQQTCEECSTLFGEYYCNICHLFDKDKKQY QLDRFKVKEVQCINCEKLQHAQQTCEDCSTLFGEYYCSICHLFDKDKRQY QLDRFKVKEVQCINCEKLQHAQQTCEDCSTLFGEYYCSICHLFDKDKKQY

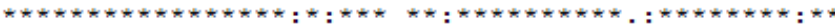

HCENCGICRIGPKEDFFHCLKCNLCLAMNLQGRHKCIENVSRQNCPICLE HCENCGICRIGPKE DF FHCLKCNLCLAMNLGRHKCIENVSRQNCPICLE HCENCGICRIGPKEDFFHCLKCNLCLAMNLQGRHKCIENVSRQNCPICLE HCENCGICRIGPKE DYFHCLKCNLCLAVNLQGKHKCIENVSRQDCPICLE HCENCGICRIGPKEDF FHCLKCNLCLAMNLQGKHKCIENVSRQNCPICLE HCENCGICRIGPKE DF FHCLKCNLCLAMNLQGKHKCIENVSRQNCPICLE HCENCGICRIGPKE DFFHCLKCNLCLAMNLQGKHKCIENVSRQNCPICLE HCENCGICRIGPKEDF FHCLKCNLCLAMNLQGKHKCIENVSRQNCPICLE HCESCGICRIGPKE DF FHCLKCNLCLTTNLRGKHKCIENVSRQNCPICLE HCESCGICRIGPKEDF FHCLKCNLCLAMTLRGKHKCIENVSRQNCPICLE

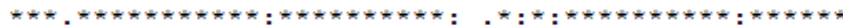

DIHTSRVVAHVLPCGHLLHRTCYEEMLKEGYRCPLCMHSALDMTRYWRQL DIHTSRVVAHVLPCGHLLHRTCYEEMLKEGYRCPLCMHSALDMTRYWRQL DIHTSRVVAHVL PCGHLLHRTCYEEMLKEGYRCPLCMHSALDMTRYWRQL DIHTSRIVARVLPCGHLLHKTCYEDMLKEGYRCPLCMHSALDMSRHWRQL DIHTSRIAAQVLPCGHLLHRTCYEDMLKEGYRCPLCMRSALDMSRSWRQR DIHTSHIVAHVL PCGHLLHRTCYEEMLKEGYRCPLCMHSALDMTRYWRQI DIHTSRVVAHVLPCGHLLHRTCYEEMLKEGYRCPLCMHSALDMTRYWRQL DIHTSRVVAHVLPCGHLLHRTCYEEMLKEGYRCPLCMHSALDMTRYWRQL DIHTSRVVAHVL PCGHLLHRTCYEEMLKEGYRCPLCMHSALDMTRYWRQI DIHTSRVVAHVLPCGHLLHRTCYEEMLKEGYRCPLCMHSALDMTRYWRQL

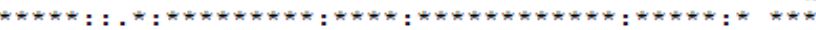

DDEVAQT PMPSEYQNMTVDILCNDCNGRSTVQFHILGMKCKICESYNTAQ DDEVAQT PMPSEYQNMTVDI LCNDCNGRSTVQFHILGMKCKICESYNTAQ DDEVAQT PMPSEYQNMTVDILCNDCNGRSTVQFHILGMKCKICESYNTAQ DDEVAQT PMPSEYQNMTVDI LCNDCNGRSTVQFHILGMKCNICESYNTTQ DDEVAQT PMP SEYQNMTVDILCNDCNGRSTVQFHILGMKCNICESYNTAQ DDEVAQT PMP SEYQNMTVDI LCNDCNGRSTVQFH I LGMKCNICESYNTAQ DDEVAQT PMP SEYQNMTVDI LCNDCNGRSTVQFH I LGMCNICESYNTAQ DDEVAQT PMP SEYQNMTVDI LCNDCNGRSTVQFH I LGMKCKI CDSYNTAQ DTEVAQT PMPSEYQNVTVDILCNDCNGRSTVQFHILGMKCKLCDSYNTAQ DIEVAQT PMPSEYQNVTVDILCNDCNGRSTVQFHILGMKCKLCDSYNTAQ $* * * * * * * * * * * * * * ;: * * * * * * * * * * * * * * * * * * * * * * * * ;: * ; * * * * ; *$

AGGRRISLDQQ

AGGRRISLDQQ

AGGRRISLDQQ

AGRYKISLDQQ

AGKYRISIDQQ

AGGCRISLDQQ

AGGCRISLDQQ

AGGRRISLDHQ

AGGRRVPVDQQ

AGGRTVSMDEQ

Fig. 3: The alignment of the protein encoded by sheep RCHY1 gene and nine other kinds of RCHY1 proteins 


\section{J. Anim. Vet. Adv., 11 (2): 252-259, 2012}

African clawed frog Western clawed frog Sheep

Cattle

Giant panda

Horse

Dog

Mouse

Rabbit

Chimpanzee

Human

Chicken

Zebrafish

African clawed frog Western clawed frog

Sheep

Cattle

Giant panda

Horse

Dog

Mouse

Rabbit

Chimpanzee

Human

Chicken

Zebrafish

African clawed. frog Western clawed frog Sheep

Cattle

Giant panda

Horse

Dog

Mouse

Rabbit

Chimpanzee

Human

Chicken

Zebrafish

African clawed frog

Western clawed frog

Sheep

Cattle

Giant panda

Horse

Dog

Mouse

Rabbit

Chimpanzee

Human

Chicken

Zebrafish

African clawed frog

Western clawed frog

Sheep

Cattle

Giant panda

Horse

Dog

Mouse

Rabbit

Chimpanzee

Human

Chicken

Zebrafish
MSKSLKKIVEESREKNVPDIDMCDRGIANMLDVPGLFTLSHITQLILSHNKLTTVPPNIA MSKSLKKIVEESREKNVPDI DMCDRGIANMLDVPGLFTLSHITQLILSHNKLTTVPPNIA MSKSLKKLVEESREKNQPEVDMSDRGISNMLDVNGLFTLSHITQLVLSHNKLTTVP PNIA MSKSLKKLVEESREKNQPEVDMSDRGISNMLDINGLFTLSHITQLVLSHNKLTTVPPNIA MSKSLKKLVEESREKNQPEVDMSDRGISNMLDVNGLFSLSHITQLVLSHNKLTTVPANIA MSKSLKKLVEESREKNQPEVDMSDRGISNMLDVNGLFSLSHITQLVLSHNKLTTVPANIA MSKSLKKLVEESREKNQPEVDMSDRGISNMLDVNGLFSLSHITQLVLSHNKLTTVPPNIA MSKSLKKLVEESREKNQPEVDMSDRGISSMLDVNGLFSLAHITQLVLSHNKLTTVP PNVA MSKSLKKLVEESREKNQTEVDMSDRGISNMLDVNGLFTLSHITQLVLSHNKLTTVPPNIA MSKSLKKMVEE SREKNQPEVDMSDRGISNMLDVNGLFTLSHITQLVLSHNKLTTVP PNIA MSKSLKKLVEESREKNQPEVDMSDRGISNMLDVNGLFTLSHITQLVLSHNKLTMVPPNIA MSKSLKKIVEESREKNQPEVDMCDRGISNMLDVPGLFTLSHITQLVLSHNKLTTVPANIA MSKSLKKIVEESRDKNLPEVDMCDRGISNLLDI PGLFSLSSITQLVLSHNKLSAVPPNIA

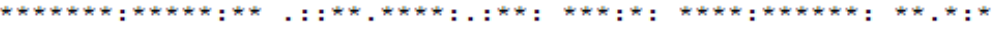

DLKNLEVLNFFNNQIEELPTQISSLQKLKHLNLGMNRLNGLPRGFGSLPALEVLDLTYNN DLKNLEVLNF FNNQIEELPTQISSLQKLKHLNLGMNRLNSLPRGFGSLPALEVLDLTYNN ELKNLEVLNF FNNQIEELPTQISSLQKLKHLNLGMNRLNTLPRGFGSLPALEVLDLTYNN ELKNLEVLNF FNNQIEELPTQISSIQKLKHLNLGMNRLNTLPRGFGSLPALEVLDLTYNN ELKNLEVLNF FNNQIEELPTQISSLQKLKHLNLGMNRLNTLPRGFGSLPALEVLDLTYNN ELKNLEVLNF FNNQIEELPTQISSLQKLKHLNLGMNRLNTLPRGFGSLPALEVLDLTYNN ELKNLEVLNF FNNQIEELPTQISSLQKLKHLNLGMNRLNTLPRGFGSLPALEVLDLTYNN ELKNLEVLNF FNNQIEELPTQISSLQKLKHLNLGMNRLNTLPRGFGSLPALEVLDLTYNN ELKNLEVLNF FNNQIEELPTQISSLQKLKHLNLGMNRLNTLPRGFGSLPALEVLDLTYNN ELKNLEVLNF FNNQIEELPTQISSLQKLKHLNLGMNRLNTLPRGFGSLPALEVLDLTYNN ELKNLEVLNFFNNQIEELPTQISSIQKLKHLNLGMNRLNTLPRGFGSLPALEVLDLTYNN DLRNIEVLNF FNNQIEELPTQISSLQKLKHLNLGMNRLNTLPRGFGSLPALEVLDLTYNN DLKNLEVLMMFNNQIEELPTQISSIQKLKHLNLGMNRLSTLPRGFGSLPALEVLDLTYNN

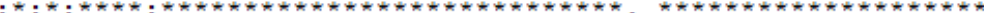

LNENSLSGNFFYLTTLRALYLSDNDFETLPPDIGKLTKLQIISLRDNDLISLPKEVGELT MNENSLPGNFFYLTTLRALYLSDNDFE ILPPDIGKLTKLQI ISLRDNDL ISLPKE IGDLT LNENSLPGNFFYLTTLRALYLSDNDFE ILPPDIGKLTKLQILSLRDNDLISLPKEIGELT LNENSLPGNFFYLTTLRALYLSDNDFE ILPPDIGKLTKLQILSLRDNDI ISLPKE IGEIT LSENSLPGNFFYLTTLRALYLSDNDFEILPPDIGKLTKLQILSLRDNDLISLPKEIGELT LNESSLPGNFFYTTLRALYLSDNDFEILPPDIGKLTKLQILSLRDNDLISLPKEIGELT LNENSLPGNFFYLTTLRALYLSDNDFE ILPPDIGKLTKLQILSLRDNDLISLPKE IGELT LNEHSLPGNFFYLTTRALYLSDNDFE ILPPDIGKLTKLQILSLRDNDLISLPKEIGELT LNENSLPGNFFYLTTLRALYLSDNDFE ILPADIGKLTKLQILSLRDNDLISLPKEIGELT LSENSLPGNFFYLTTLRALYLSDNDFE ILPPDIGKLTKLQILSLRDNDLISLPKE IGELT LSENSLPGNFFYLTTLRALYLSDNDFE ILPPDIGKLTKLQILSLRDNDLISLPKEIGELT LNENSLPGNFFYLTTLRALYLSDNDFE ILPPDIGKLTKLQILSLRDNDLISLPKE IGELT LNESSLPGNFFYLTTLRALYLSDNDFEILPPDIGKLAKLQILSLRDNDLISLPREIGDLT

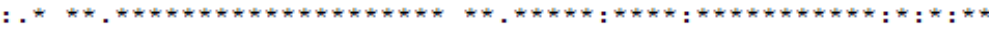

QLKELHIQGNRLTVLPPELGNLDLTGQKQVFKAENNPWVT PIADQFQVGVSHVFEYIRSE QLKELHIQGNRLTVLPPELGNLDLTGQKQVFKAENNPWVT PIADQFQVGVSHVFEYIRSE QLKELHIQGNRLTVLPPELGNLDLTGQKQI FKAENNPWVT PIADQFQLGVSHVFEYIRSE QLKELHIQGNRLTVLPPELGNLDLTGQKQI FKAENNPWVT P IADQFQLGVSHVFEYIRSE QLKELHIQGNRLTVLPPELGNLDLTGQKQVFKAENNPWVT PIADQFQLGVSHVFEYIRSE QLKELHIQGNRLTVLPPELGNLDLTGQKQVFKAENNPWVT PIADQFQLGVSHVFEYIRSE QLKELHIQGNRLTVLPPELGNLDLTGQKQVFKAENNPWVT PIADQFQLGVSHVFEYIRSE QLKELHIQGNRLTVLPPELGNLDLTGQKQVFKAENNPWVT PIADQFQLGVSHVFEYIRSE QLKELHIQGNRLTVLPPELGNLDLTGQKQVFKAENNPWVT PIADQFQLGVSHVFEYIRSE QLKELHIQGNRLTVLP PE LGNLDLTGQKQVFKAENNPWVT P IADQFQLGVSHVFEYIRSE QLKELHIQGNRLTVLPPELGNLDLTGQKQVFKAENNPWVT PIADQFQLGVSHVFEYIRSE QLKELHIQGNRLTVLPPELGNLDLTGQKQVFKAENNPWVT PIADQFQLGVSHVFEYIRSE QLKELHIQGNRLTVLPPELGNLDLTGPKQVFKAENNSWVT PIADQFQLGISHVFEYIRSE

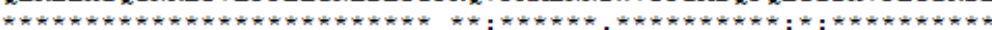

TYKYLYGRHMQANPE P PKKNNDKSKKISRKPLAAKNK TYKYLYGRHMQANPE P PKKNNDKSKKISRKPLAAKNK TYKYLYGRHMQANPE PPKKNNDKSKRISRKPLAAKNK TYKYLYGRHMQANPE P PKKNNDKSKKISRKPLAAKNK TYKYLYGRHMQANPE P PKKNNDKSKKISRKPLTAKNK TYKYLYGRHMQANPEP PKKNNDKSKKISRKPLTAKNK TYKYLYGRHMQANPE P PKKNNDKSKKISRKPLTAKNK TYKYLYGRHMQANPE P PKKNNDKSKKISRKPLAAKNK TYKYLYGRHMQANPE P PRKNNDKSKKISRKPLTAKNK TYKYLYGRHMQANPE P PKKNNDKSKKISRKPLAAKKNR TYKYLYGRHMQANPEP PKKNNDKSKKISRKPLAAKNR TYKYLYGRHMQANPE P PKKNNDKSKKISRKPLAARNK TYKYLYGRHMQANPEAPKKNADKSKKISRKPLAIAKNK
$* * * * * * * * * * * * * * *, *: * * * * * *: * * * * * *: *: *:$

Fig. 4: The alignment of the protein encoded by sheep RSU1 gene and twelve other kinds of RSU1 proteins 


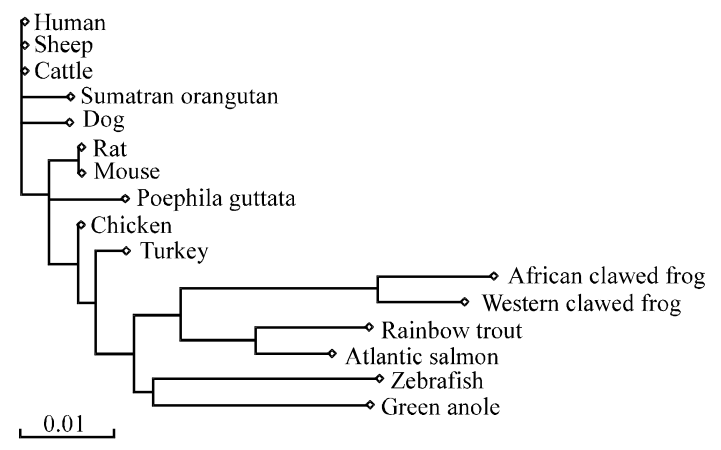

Fig. 5: The phylogenetic analysis for sixteen kinds of $R H O A$ genes

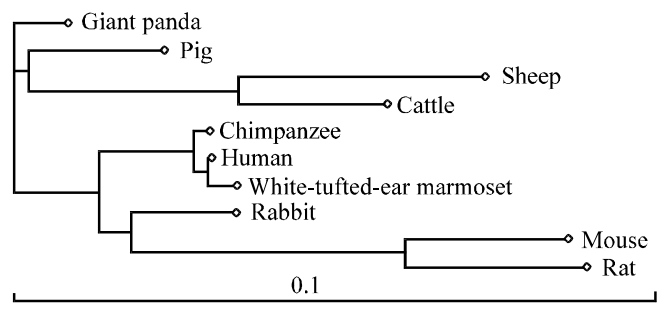

Fig. 6: The phylogenetic analysis for ten kinds of $R C H Y 1$ genes

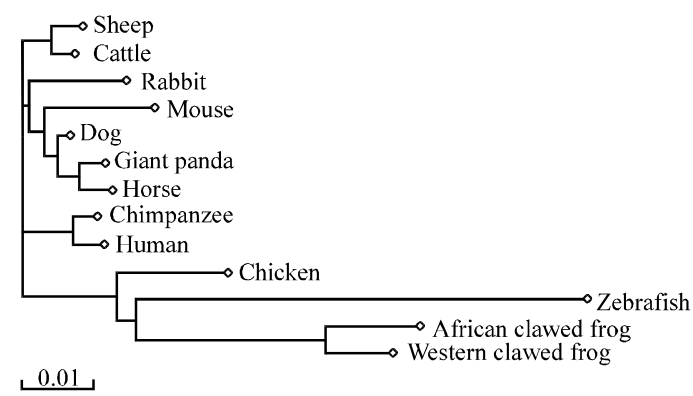

Fig. 7: The phylogenetic analysis for thirteen kinds of RSU1 genes

genes. Based on these sheep EST sequences, the researchers can obtain the complete coding sequences of some novel sheep genes through the some experimental methods such as RT-PCR. From the clone and sequence analysis of sheep RHOA, RCHY1 and RSU1 genes, it could be seen that this is an effective method to isolate some novel sheep genes.

Through sequence analysis, the researchers found that the encoding protein of the sheep $R H O A, R C H Y 1$ and RSU1 genes are highly homologous with RHOA, RCHY1 and RSU1 proteins of human and some other animals. This implied that the RHOA, RCHY1 and RSU1 genes were highly conserved in some species and the sheep $R H O A, R C H Y 1$ and RSU1 genes will have similar

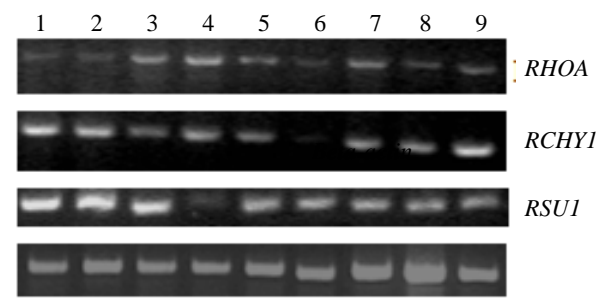

Fig. 8: Tissue expression distribution of sheep RHOA, RCHY1 and RSU1 genes. The beta-actin expression is the internal control. 1 : muscle; 2 : heart; 3: lung; 4: spleen; 5: skin; 6: fat; 7: liver; 8: kidney; 9: ovary

functions as the RHOA, RCHY1 and RSU1 genes of human and other animals. The researchers also found that the sheep RHOA, RCHY1 and RSU1 proteins do not show complete identity to some animals. This implied that the sheep RHOA, RCHY1 and RSU1 genes will have some differences in functions to those of other animals.

The phylogenetic analysis revealed that the sheep $R H O A, R C H Y 1$ and $R S U 1$ genes all have a closer genetic relationship with the RHOA, RCHY1 and RSU1 genes of cattle. This implied that we can use cattle as a model organism to study the sheep RHOA, RCHY1 and RSU1 genes or use sheep as a model organism to study the cattle RHOA, RCHY1 and RSU1 genes.

From the tissue distribution analysis in the experiment, it can be seen that the sheep RHOA, RCHY1 and $R S U 1$ genes were obviously differentially expressed in some tissues. As the researchers did not study functions at protein levels yet there might be many possible reasons for differential expression of sheep $R H O A, R C H Y 1$ and $R S U 1$ genes. The suitable explanation for this under current conditions is that at the same time those biological activities related to the mRNA expression of sheep RHOA, RCHY1 and RSU1 genes were presented diversely in different tissues.

\section{CONCLUSION}

In this study, the researchers first isolated the sheep $R H O A, R C H Y 1$ and $R S U 1$ genes and performed necessary sequence and tissue expression profile analysis. This established the primary foundation for further insight into these novel sheep genes.

\section{ACKNOWLEDGEMENTS}

This research was supported by the Candidates of the Young and Middle Aged Academic and Technical Leaders of Yunnan province. 


\section{REFERENCES}

Chunduru, S., H. Kawami, R. Gullick, W.J. Monacci, G. Dougherty and M.L. Cutler, 2002. Identification of an alternatively spliced RNA for the Ras suppressor RSU-1 in human gliomas. J. Neurooncol., 60: 201-211.

Dougherty, G.W., C. Jose, M. Gimona and M.L. Cutler, 2008. The Rsu-1-PNCH1-LK complex is regulated by Ras activation in tumor cells. Eur. J. Cell Biol., 87: $721-734$.

Dougherty, G.W., T. Chopp, S.M. Qi and M.L. Cutler, 2005. The Ras suppressor Rsu-1 binds to the LIM 5 domain of the adaptor protein PINCH1 and participates in adhesion-related functions. Exp. Cell Res., 306: 168-179.

Liu, G.Y. and S.Z. Gao, 2009. Molecular cloning, sequence identification and tissue expression profile of three novel sheep (Ovis aries) genes-BCKDHA, NAGA and HEXA. Biol. Res., 42: 69-77.

Liu, G.Y., 2009. A novel HADHA gene differentially expressed in muscle and other tissues from blackboned vs. ordinary sheep. Anim. Sci. Pap. Rep., 27: $127-137$.

Liu, G.Y., Y.Z. Xiong, C.Y. Deng, B. Zuo and J.H. Zhang, 2004. Comparison of gene expression patterns in Longissimus dorsi of pigs between the high-parent heterosis cross combination Landrace $\times$ Large White and the mid-parent heterosis cross combination Large White $\times$ Meishan. Asian-Aust. J. Anim. Sci., 17: 1192-1196.

Maekawa, M., T. Ishizaki, S. Boku, N. Watanabe and A. Fujita et al., 1999. Signaling from Rho to the actin cytoskeleton through protein kinases ROCK and LIM-kinase. Science, 285: 895-898.
Ohta, Y., N. Suzuki, S. Nakamura, J.H. Hartwig and T.P. Stossel, 1999. The small GTPase RalA targets filamin to induce filopodia. Proc. Natl. Acad. Sci. USA., 96: 2122-2128.

Peyton, S.R., P.D. Kim, C.M. Ghajar, D. Seliktar and A.J. Putnam, 2008. The effects of matrix stiffness and RhoA on the phenotypic plasticity of smooth muscle cells in a 3-D biosynthetic hydrogel system. Biomaterials, 29: 2597-2607.

Saeki, N., H. Tokuo and M. Ikebe, 2005. BIG1 is a binding partner of myosin Ixb and regulates its RhoGTPase activating protein activity. J. Biol. Chem., 280: 10128-10134.

Shloush, J., J.E. Vlassov, I. Engson, S. Duan and V. Saridakis et al., 2011. Structural and functional comparison of the RING domains of two p53 E3 ligases, Mdm2 and Pirh2. J. Biol. Chem., 286: 4796-4808.

$\mathrm{Su}$, W., P. Chardin, M. Yamazaki, Y. Kanaho and G. Du, 2006. RhoA-mediated phospholipase D1 signaling is not required for the formation of stress fibers and focal adhesions. Cell Signal, 18: 469-478.

Wu, G., M. Sun, L. Zhang, J. Zhou, Y. Wang and K. Huo, 2010. A novel hPirh2 splicing variant without ubiquitin protein ligase activity interacts with p53 and is down-regulated in hepatocellular carcinoma. FEBS Lett., 584: 2772-2778.

Yan, J., D. Zhang, Y. Di, H. Shi, H. Rao and K. Huo, 2010. A newly identified Pirh2 substrate SCYL1-BP1 can bind to MDM2 and accelerate MDM2 selfubiquitination. FEBS Lett., 584: 3275-3278.

Yonggang, L. and G. Shizheng, 2009. A novel sheep gene, MMP7, differentially expressed in muscles from black-boned sheep and local common sheep. J. Applied Genet., 50: 253-256. 